\title{
Summary Talk: Quantum Field Theory
}

\author{
C. A. A. de Carvalho \\ Instituto de Física, Universidade Federal do Rio de Janeiro, Caixa Postal 68528, 21941-972, Rio de Janeiro, RJ, Brasil
}

Received on 22 August, 2003.

We present a summary of the contributions to Quantum Field Theory of the XXIII Brazilian National Meeting on Particles and Fields.

Quantum Field Theory (QFT) arrived in Águas de Lindóia as a rather successful theory in her early seventies, still charming, rich, and powerful. Its many admirers certainly include most of the participants in the meeting, for reasons which we will try to highlight.

Born of the marriage of two fundamental constants of Nature, Planck's $h$, from Quantum Mechanics, and the speed of light $c$, from Special Relativity, it grew up on two basic ingredients: quantization and symmetry. Notoriety came as early as the 1940's, with the success of Quantum Electrodynamics (QED), thanks to the Nobel winning work of Feynman, Schwinger, and Tomonaga, and to the seminal contributions of many other historic names, among which that of Dyson certainly deserves a special place. The late 1940's, and the 1950's, witnessed the infancy of developments that would play a crucial role in the years to come: path-integrals, functional methods, and the renormalization group, on the quantization side, as well as space-time and gauge symmetries, led to important advances that would fully blossom more than a decade afterwards. It is true that in the 1960's QFT had to face many challenges, but it survived them all, and by the end of the decade was ready to produce a series of remarkable achievements.

Electroweak unification (1967), Quantum Chromodynamics (QCD)(1972), the renormalization of gauge theories (1971,1972), asymptotic freedom (1973), semiclassical methods based on topological solutions (1974), and nonperturbative calculations in lattice gauge theories $(1974,1979)$ paved the way for establishing the Standard Model of Strong and Electroweak interactions as the best description of the world of particles and fields up to $\mathrm{TeV}$ energies. However, in the 1970's, the need to go to even higher energies led, on the symmetry side, to the study of larger gauge groups $\left(S U(5), S O(10), E_{6}\right)$ in search of a grand-unification of strong and electroweak forces. In that same decade, both quantization and symmetry had to deal with graded algebras for symmetry generators, as supersymmetry and superspace entered the scene. Also, extended objects reappeared in the 1970 's in a different context, and were pursued in the 1980's as a viable alternative to the ultimate unification of all forces, and to the quantization of gravity: superstrings in higher di- mensions, the Conformal Field Theories (CFT's) on their worldsheets, and the compactifications aimed at extracting gauge symmetries out of space-time became the subject of intense investigation.

The 1990's were marked by the need to extend the theory beyond its usual environments: i) beyond local fields, as strings and branes played a central role in attempts at unification with gravity, leading to expected (at low-energies), and unexpected (AdS/CFT bulk-boundary) connections with field theories, to superstring field theories, extra dimensions, dualities that point to an underlying $\mathrm{M}$ (ysterious)- or M(agic)-theory, and noncommutative geometries; ii) beyond the flat space vacuum, as required to describe external fields or boundaries (as in the Casimir effect), curved spaces (as in the semiclassical descriptions of the Hawking and Fulling-Davies-Unruh effects), and cosmological models of inflation; iii) into Condensed Matter and Statistical Mechanics, as illustrated by the field-theoretic descriptions of polymers, quantum Hall effect, high- $T_{c}$ superconductivity, and strongly correlated systems; by the conformal field theory descriptions of $2 D$ critical phenomena; by the application of field theory methods to the study of phase transitions, as in Bose-Einstein condensation; iv) into environments at finite temperatures and particle densities, to describe conjectured new phases of matter such as quark-gluon plasmas, disoriented chiral condensates (DCC), and color superconductors in QCD; v) beyond the Standard Model, in order to test its supersymmetric extensions with experimental data to come from future colliders.

Viewed in an extended context, to include the many advances of String Theory that were inspired by or connected with it, QFT is quite alive, and foresees the future with high hopes of both unifying the forces of Nature, and amplifying its realm of applications. Clear evidence of this positive attitude could be seen in the fourth edition of the big conference on Theoretical Physics first held in Japan in 1953, which took place in Paris, in July, under the name TH-2002. Of the plenary talks, eight out of seventeen were connected with QFT: Probing Physics at the Planck Scale (G. Ross), Conformal Invariance in Percolation, SAW's, and Related Problems (J. Cardy), QCD and Natural Phylosophy (F. Wilczek), AdS/CFT and dS/CFT (A. Ströminger), Tachyons in String Theory (A. Sen), Lattice QCD (M. Lüscher), Inflationary 
Cosmology (A. Linde), and Three Melodies of XXth Century Theoretical Physics (C. N. Yang). The latter was the closing talking, in which quantization, symmetry, and phase factor (gauge symmetry) were the three tunes Yang referred to in his title.

The XXXIII Brazilian Meeting on Particles and Fields also had an impressive number of contributions in Quantum Field Theory. Three of the six plenary talks were dedicated to subjects of current interest in the area: Electroweak Symmetry Breaking (R. Barbieri), Strings and Large- $N$ Limit of Gauge Theories (J. M. Maldacena), and Gauge Theories on the Light Front (S. Brodsky). There were six, out of eleven, parallel talks that dealt with QFT. Three were held in QFT sessions: Topological Mass Generation in a Supersymmetric World with R-Parity (C. Wotzasek), Aspects of Dimensionality and Finiteness Properties of Supersymmertic Field Theories (J. Helayel), and Covariant Formalism for the Superstring (N. Berkovits). Three others were held in sessions on Cosmology and Gravitation, and Phenomenology: Elementary Particles under the Lens of Blackholes (G. A. Matsas), New Useful Gauge Extension of the Supersymmetric Standard Model (E. Ma), and The Search for the Quark-Gluon Plasma: 20 years later (T. Kodama). Besides, there were 47 oral communications, and 111 posters on [1]:

i The world of strings and branes;

ii Conformal field theories;

iii Noncommutative field theories;

iv Supersymmetric extensions;

v QFT at finite temperature and/or density;

vi QFT in various backgrounds (semiclassical);

vii Classical field theories; viii Nonperturbative calculations;

ix Integrable models;

$\mathrm{x}$ Mathematical aspects of quantization and symmetry;

xi Applications (Bose-Einstein condensates, magnetic systems, Hall systems, strongly correlated systems).

The list above shows that the meeting covered practically all topics of present interest in QFT. Several of the presentations were the fruit of scientific collaborations, many among them involving graduate students. There were many discussions during the various sessions, held in a very pleasant and lively atmosphere, which has become a trademark of the Particles and Fields area.

Quantum Field Theory was a paradigm for XXth century Physics. From what was presented in the meeting, one may hope that its extensions may lead to unification, as well as to its widespread use in several other areas of Physics and beyond. Wider usage will certainly promote a richer interplay with phenomenology, more experimental checks, and wider acceptance as a basic tool in science. Águas de Lindóia provided a stimulating proof that, as far as QFT is concerned, life begins at seventy, and that much more is yet to be accomplished in the years to come.

\section{Acknowledgments}

The author acknowledges support from $\mathrm{CNPq}$ and FUJB/UFRJ.

\section{References}

[1] Program and Abstracts, XXXIII Brazilian National Meeting on Particles and Fields, October 15-19, 2002, edited by Sociedade Brasileira de Física (SBF). 